\title{
HISTORIA DE UN TRADUCTOR, PROLOGUISTA Y ANOTADOR: TYNDALE Y LOS PRIMEROS PASOS DE LA BIBLIA EN INGLÉS
}

\author{
J.J. LANERO \\ Universidad de León
}

\begin{abstract}
Resumen
El tema de nuestro estudio es la labor traductora de William Tyndale. En Alemania y en los Países Bajos, entre 1520 y 1540, tradujo al inglés el Nuevo Testamento del griego, dos veces; la mitad del Antiguo Testamento, del hebreo, y publicó sus traducciones en pequeños libros de bolsillo que, a modo de contrabando, se introducían en Inglaterra.

Tyndale se sirve de los textos, en paralelo, griegos y latinos de Erasmo; del Nuevo Testamento de Lutero, de la traducción francesa del Nuevo Testamento y de las Escrituras en hebreo. La obra de Tyndale pasó a la edición revisada de Coverdale de 1539, la denominada Gran Biblia, y de ésta a la Biblia de Ginebra de 1560 y sus ediciones posteriores. Así hasta llegar a la mesa de los traductores designados por el rey James en 1607, que la adoptaron en su mayor parte. De esta suerte, la Biblia del King James, durante cuatro siglos, ha sido sin decirse, obra de Tyndale.

Palabras claves: Antiguo Testamento, Nuevo Testamento, griego, hebreo.
\end{abstract}

\begin{abstract}
The topic of this paper is William Tyndale's translations. Either in Germany or in The Netherlands, between 1520 and 1540, he translated the New Testament from Greek into English, twice; half of the Old Testament, from the Hebrew, and published his translations in small pocket books which were smuggled into England.

Tyndale uses Erasmus' texts in Greek and Latin, in parallel; Luther's New Testament; the French translation of the New Testament and the Scriptures in Hebrew. Tyndale's work went into Coverdale's revised edition of 1539, the Great Bible, and from it into the Geneva Bible of 1560 and its succeeding editions until reaching the table of the translators appointed by the King James in 1607, who broadly adopted it. In so doing, the King James Bible has been, without being said, Tyndale's work.

Key words: Old Testament, New Testament, Greek, Hebrew.
\end{abstract}


íos, mares tal vez, de tinta se han empleado en los análisis de las
diversas traducciones inglesas de la Biblia. La Reforma que, fuera de toda duda, genera una controversia en la identidad espiritual y creyente de Inglaterra, es algo más que la caricatura del capricho del monarca para conseguir la nulidad matrimonial. La jerarquía eclesiástica, es posible que para conservar más su status temporal que la salud espiritual de sus fieles, fue durante mucho tiempo estricta con la circulación de la Biblia en inglés, temerosa de la libre interpretación de los fieles. La iglesia, poco a poco, se fue modificando, sobre todo a lo largo del reinado de Isabel I. Con el inicio de la dinastía de los Estuardo, y con todas las bendiciones de monarca reinante, reclamando la colaboración de todos los centros del saber de la época, se acometió la monumental tarea que se iba a substanciar en la denominada King James Bible, de 1611.

Con cierta frecuencia, excesiva quizás, los estudiosos bíblicos se han concentrado en este texto olvidando la labor meritoria de los traductores que lo precedieron, muy a pesar de que sus versiones todavía respiran por el texto de 1611.

El objetivo de este estudio, breve $y$, por lo tanto, necesariamente incompleto, es seguir los pasos de las traducciones de William Tyndale, al que sólo la muerte le impidió presentar una Biblia completa en inglés. Sus dificultades no sólo se circunscribieron al hallazgo de una imprenta dispuesta a publicar sus versiones, en ocasiones, como en la suya, el precio fue la propia vida.

Así, hacia mediados de la década de 1520, la imprenta en Inglaterra iba muy por detrás de la del resto de Europa. Podrían citarse por miles los 
impresores europeos, de los que nos queda constancia, anteriores a 1500. Londres tenía tres. Uno, muy bueno: Caxton. Los otros dos, de Worde y Pynson, no alcanzaron, ni de lejos, la calidad de las obras de sus colegas europeos.

La escasez de imprentas, $\mathbf{u}$ otras razones añadidas, se materializa, también, en la carencia de Biblias completas en inglés. Los alemanes contaban con ella desde 1466; catalanes, checos, franceses, holandeses e italianos, desde la década de 1470. Las traducciones españolas y portuguesas también vieron la luz antes de 1500. Todas ellas, es cierto, utilizan como texto original el latino. Cuando, en 1522, Lutero presentó el innovador Nuevo Testamento de "Septiembre", traducido del original griego, Alemania ya había conocido dieciocho ediciones distintas de la Biblia en alemán.

La iglesia inglesa tenía miedo de lo que podía significar la existencia de Biblias en inglés, obsesionada, como estaba, con las traducciones manuscritas lolardas de la Vulgata, realizadas en la década de 1380 bajo la inspiración de John Wyclif. Las constituciones de Oxford de 1407-9 prohibieron producir o poseer semejantes traducciones. Un siglo después, la posesión de ese material lolardo llevó a unos cuantos a la hoguera.

El tema de nuestro estudio es la labor de William Tyndale. En Alemania y en los Países Bajos, entre 1520 y 1540, tradujo al inglés el Nuevo Testamento del griego, dos veces; la mitad del Antiguo Testamento, del hebreo, y publicó sus traducciones en pequeños libros de bolsillo que, a modo de contrabando, se introducían en Inglaterra.

Tyndale fue un estudioso y un traductor de altura. Su obra se incorporó, casi directamente, a la King James Bible. Y no creemos exagerar si afirmamos que sigue influyendo en las traducciones bíblicas actuales. A través de la King James Bible, que dominó en el mundo angloparlante durante 350 años, Tyndale ha 
llegado hasta tanta gente como el mismo Shakespeare. Otorgó a la prosa un estilo sencillo. Precisamente en un momento, primeras décadas del siglo XVI, en el que la lengua inglesa, no despuntaba y pocos le auguraban futuro. Por todo esto, o a su pesar, las traducciones bíblicas de Tyndale no se han considerado desde la importancia que tienen.

\section{II}

Tyndale pertenecía a una familia acaudalada del condado de Gloucester. Pasó doce años en Oxford y es posible que también estuviera en Cambridge poco después de que Erasmo concluyera su tarea de profesor de griego. A su regreso a Gloucester, fue ordenado sacerdote. Poseía la versión latina de Erasmo del Nuevo Testamento, del original griego: el Novum instrumentum, de 1516.

Erasmo había impreso su traducción en bilingüe -griego y latín-, lo que hacía de su obra una contribución importante para los reformadores europeos deseosos de regresar a las raíces del Nuevo Testamento. Tyndale, a su vez, era un finísimo conocedor del griego. Ofreció su colaboración a Cuthbert Tunstall, amigo de Erasmo y obispo de Londres, que la rechazó. Después de haberse establecido en Colonia, pasó a Worms, en donde en 1526, terminó su Nuevo Testamento, del que algunas copias lograron llegar a Inglaterra. En Alemania aprendió hebreo, prácticamente desconocido en Inglaterra. En 1530, en Amberes, publicó su Pentateuco, la primera traducción que se hacía del hebreo al inglés. En 1534 revisó su Nuevo Testamento y concluyó la traducción de los libros históricos del Antiguo Testamento. En 1535 fue arrestado y encarcelado en el castillo de Vilvoorde, a las afueras de Bruselas. Permaneció allí dieciséis 
meses. En la madrugada del 6 de Octubre de 1536, en el transcurso de una pomposa ceremonia, lo estrangularon primero y quemaron después por hereje:

Tyndale (...) was formally condemned as a heretic, degraded from the priesthood and handed over to the secular authorities for punishment -that is, burning at the stake (...). In the case of a priest (...) the anointing oil was symbolically scraped from his hands, the bread and wine of the Mass placed there and removed and the vestments ceremonially stripped away. William Tyndale, suffering this, no longer a priest, would find the secular officer waiting alongside to receive him ${ }^{1}$.

III

Tyndale intentó publicar, por primera vez, una Biblia en inglés en la imprenta de Peter Quentell de Colonia, en 1525. Colonia había sido el centro impresor alemán por excelencia durante más de cincuenta años, bajo la dirección de Heinrich Quentell, que suministraba a las universidades locales los textos escolásticos. El nieto de Heinrich, Peter, se especializó también en textos de la ortodoxia católica:

Peter Quentell, Heinrich's grandson, finally became the leading antiLutheran printer after 1520, although this did not prevent him from printing for William Tyndale the Protestant English translation of the New Testament in 152452 .

Uno de los autores asociados a Peter Quentell fue el antiluterano Johannes Dobneck o Cochlaeus que, en 1525, publicaba en su imprenta De Petro et Roma, adversus Velenum Lutheranum. La estrategia que se había impuesto Cochlaeus era la de persuadir a personalidades inglesas para que escribieran prólogos

${ }^{1}$ David Daniell, William Tyndale: A Biography, New Haven \& London, Yale University Press, 1994, p. 374 .

2 S. H. Steinberg, Five Hundred Years of Printing, New Edition, Revised by John Trevitt, London, The British Library \& Oak Knoll Press, 1996, p. 28. 
para sus libros antiluteranos. Estaba muy bien relacionado con la corte inglesa. En una ocasión, bien sorprendió embriagados, bien emborrachó él mismo, a los trabajadores de la imprenta de Quentell, a los que escuchó que había dos ingleses merodeando por allí, muy educados y que dominaban varias lenguas, que estaban imprimiendo un Nuevo Testamento luterano en inglés, trabajo que se encontraba bastante avanzado:

The printing had reached as far as Matthew 22, in the middle of what would later be verse 12 , at the foot of the next page following signatura $\mathrm{H}$, when it was broken off. The Cologne authorities were about to arrest the two Englishmen and impound their work. Tyndale and Roye escaped and fled up the Rhine to Worms, taking their work with them. The agent of the interruption was a Cologne scholarwriter called John Dobneck, whose Latin name was Cochlaeus. Work of his in several large volumes was also being printed at Peter Quentell's press, and he came to know the workers in the print shop ${ }^{3}$.

Uno de esos hombres, pues, era William Tyndale y el otro, William Roye. Cochlaeus informó de lo que se había enterado y el Senado de Colonia hizo pública una prohibición contra Quentell. El propio Cochlaeus intentó incautar la obra, pero era demasiado tarde. Los dos ingleses habían huido con las hojas impresas que, posteriormente circularían por Inglaterra. Constituyen el primer documento luterano en inglés. En la actualidad se conserva un único ejemplar; se trata de un bello documento que pertenece a la Colección Grenville de la Biblioteca Británica.

El suceso impresionó tanto a Cochlaeus que dio a la estampa tres narraciones diferentes de los hechos: una en 1533, otra en 1538 y la última en una fecha tan tardía como 1549. Su definición del Nuevo Testamento de Tyndale como «luterano» es exacta. A semejanza del Nuevo Testamento de Lutero, de 1522, es un libro en cuarto, dispone de preliminares, un largo prólogo y el estilo y contenido de las notas al margen está en rotonda, a

${ }^{3}$ David Daniell, 1994, op. cit., p. 109. 
diferencia de la letra gótica del texto. En definitiva, tenemos a Lutero en inglés. En la primera página San Mateo introduce su pluma en un tintero que le sostiene un ángel.

No debemos dejar escapar un dato: que el ortodoxo Peter Quentell imprimiera esta traducción herética, arriesgando su propia vida, algo nos dice de la seguridad que tenía de las ventas en Inglaterra a pesar de que sabía que la traducción era ilegal.

Pero conviene que aludamos a las similitudes que existen con el primer Nuevo Testamento de Lutero, el denominado de «Septiembre». Debemos tener en cuenta tres aspectos: los ingleses, en 1525, desconocían la Biblia completa; dónde aprendió Tyndale hebreo y qué grado de influencia ejerció Lutero en las traducciones de Tyndale.

Está claro que los ingleses, aunque no disponían de una Biblia en su lengua, conocían algunas historias bíblicas. Sus fuentes eran los sermones, las vidrieras, los bordados de los paños sacros, los ciclos de los Mystery Plays, que cada año se representaban en York, Coventry y en algún otro lugar. Algo sabían, por lo tanto, de la creación, de Adán y Eva, del arca de Noé, de Abraham e Isaac, de Jonás y la ballena, de la anunciación, del nacimiento de Jesús, de algunas de sus parábolas, de su muerte y resurrección, de Pentecostés, del día del juicio final. Pero, por muy representativos que sean todos estos pasajes, no constituyen, ni con mucho, más que unos pocos fragmentos bíblicos, pues estaba prohibido conocerla entera. Ante las reivindicaciones de la gente, el Canciller Tomás Moro sugiere que pequeños fragmentos, por ejemplo, la mitad del libro de Josué, se podían permitir a ciertas personas. No obstante, pendía la amenaza de la pena de muerte para aquellos que se hicieran con todos los fragmentos para así poder tener una Biblia completa en inglés:

...it myght be wyth dylygence well and truely translated by some good catholyque 
and well lerned manne / or by diuers dyuydynge the laboure amonge theym / and after conferrynge theyr seuerall partys togyther eche with other. And after that might the worke be allowed and approued by the ordynaryes / and by theyr authorytees so put vnto prente / as all the copyes sholde come hole vnto the byshoppys hande. Whiche he may after hys dyscrecyon and wysdome delyuer to suche as he perceyueth honeste sad and virtuous / with a good monycyon \& fatherly counsayll / to vse it reuerently with humble hart \& lowly mynde / rather sekyng therin $\left[\mathrm{X}_{4} \mathrm{~V}\right]$ occasion of deuocyon than of dyspycyon. And prouydyng as moche as may be / that the boke be after the deceace of the partye brought agayne and reuerently restored vnto the ordinary ${ }^{4}$.

Está claro que Moro y la jerarquía eclesiástica no deseaban que el pueblo leyera la Biblia para que no se hicieran una idea errónea: ese sería el caso de la justificación por la fe y no por las obras, según dice la Carta a los Romanos, 5, 7 y 8. Esta doctrina necesita de la Biblia completa, pues la Biblia sólo se comprende en su totalidad. Continúa siendo un misterio, todavía hoy, cómo era posible que la iglesia inglesa estuviera dispuesta a enviar a la hoguera a cualquiera que poseyera un fragmento de la Biblia lolarda. El pueblo reclamaba una Biblia en su lengua, como lo demuestra el gran riesgo que corrían los impresores alemanes y flamencos de Biblias inglesas. A esto debemos añadir la existencia de grupos de lectura de la Biblia en pueblos y ciudades de Inglaterra, sin olvidar los relatos de las inquisiciones de hombres y mujeres del pueblo llano formuladas por eclesiásticos que se quedaban estupefactos ante el amplio y profundo conocimiento bíblico de aquellas gentes que, en algunos casos, no sabían ni leer. En efecto. Así sucedió cuando, gracias a Tyndale, la Biblia en inglés se convirtió en algo accesible. Desde el final del reinado de Enrique VIII, en 1547, hasta, prácticamente, nuestros días, el pueblo inglés ha estado en permanente vínculo con la Biblia.

4Thomas More, A Dialogue Concerning Heresies, en Thomas M.C. Lawler, Germain Marc'hadour and Richard C. Marius (eds.), The Complete Works of St. Thomas More, New Haven and London, Yale University Press, 1976, vol. VI, Part I, p. 341. 
Tyndale fue un destacado intelectual. Los hay que piensan que, incluso, mejor que Erasmo. Manejaba con destreza el latín humanista que había aprendido en Oxford. Y lo mismo puede decirse del griego. Como prueba de su conocimiento, presentó a Cuthbert Tunstall su traducción de una oración de Isócrates. Sabemos por Buschius, intelectual alemán contemporáneo, que hablaba ocho lenguas. Tyndale tradujo del alemán, español, francés, griego, hebreo, italiano y latín. Lo que continúa siendo un misterio es dónde aprendió hebreo. Hay que descartar Inglaterra, pues, aunque la idea de los colegios trilingües (latín, griego y hebreo) era corriente, en aquel momento sólo dos personas en Cambridge sabían hebreo y ninguna estaba interesada en la traducción. No parece verosímil que Tyndale estudiara hebreo con Robert Wakefield, el primer hebraista inglés, que regresó a Cambridge de Lovaina en 1520.

Resulta más creíble que Tyndale estudiara hebreo en Alemania, donde existían escuelas rabínicas en varias ciudades, como Worms, en la que residió. Johannes Reuchlin había publicado su gramática hebrea y el diccionario De rudimentis hebraicis (1506). Reuchlin se encontró en una situación difícil con los eclesiásticos, pues mantenían que la Biblia original estaba escrita en latín. Resulta sorprendente comprobar que no recibió el apoyo de Erasmo. Es posible que Tyndale adquiriera una Biblia hebrea en Alemania. Le hubiera sido de mucho beneficio la Biblia Políglota Complutense, publicada en Alcalá de Henares en 1522, pues habría puesto en sus manos la Biblia hebrea, los textos latino y griego comparados, una gramática y un diccionario, además de los Targums, es decir, los comentarios y paráfrasis en arameo.

Para acceder a esta obra espléndida, hubiera necesitado una biblioteca 
universitaria. Durante años corrió la especie de que Tyndale había pasado algún tiempo con Lutero en la Universidad de Wittenberg. No hay prueba documental al respecto, aunque su biógrafo Mozley afirma haberlo encontrado en los archivos de Wittenberg bajo el nombre de «Daltici»:

In the registers of the university of Wittenberg we read that Guilhelmus Roy ex Londino, William Roye of London, matriculated on June 10, 1525, more than a year after Tyndale's departure from England (...). A year earlier a name meets our eyes (...). This is Matthias von Emersen of Hamburg (...) who entertained Tyndale five years later, when he visited that city. This young man matriculated on May 30, 1524; and in close neighbourhood to him, under date May 27, stands the name Guillelmus Daltici ex Anglia, William Daltici from England. Who is this?

Its form seems not at home in English, or Latin, or in any other tongue that Tyndale was likely to use. But suddenly it flashed upon me that by reversing the two syllables of Tindal you get Daltin, which only differs from Daltici by one letter. The present register is but a copy of the original, and if the copyist misread the final letter, all becomes clear. In those perilous times it was common enough for men to disguise their names ${ }^{5}$.

El rumor de que era un cómplice de Lutero lo extendieron sus enemigos en Inglaterra como calumnia política. Por otra parte, parece estar clara la inicial dependencia de Tyndale de la traducción que Lutero había hecho del Nuevo Testamento así como de sus prólogos.

Todo parece indicar que Tyndale aprendió hebreo entre 1526 y 1530. Cuando imprimió su Nuevo Testamento revisado, en 1534, comenzó su Prólogo en el que incorporó explicaciones de los cambios que había realizado. Había conocido la frase hebrea y la impronta que deja en las palabras griegas. Este extremo, generalmente aceptado por los especialistas en el Nuevo Testamento, lo difundió Tyndale, a la vez que nos hace pensar que aprendió hebreo antes de comenzar la traducción del Nuevo Testamento.

Publicó el Pentateuco en Amberes en 1530, en donde residía desde 1528, fecha en la que aparecieron sus primeros libros doctrinales. 
El fragmento que se imprimió en Colonia, en 1525, se ha descrito como «Lutero en inglés». Cualquiera que conozca la edición espléndida de Lutero de 1522, puede comprobar la similitud. Todo ello a pesar de que el libro de Tyndale sea un pequeño volumen en cuarto, mientras que el de Lutero es un elegante folio. No obstante tiene la misma disposición de páginas, parecidos grabados, textos al margen muy paralelos y una lista introductoria de libros del Nuevo Testamento que es idéntica en orden y disposición. Desde San Mateo al Tercer Evangelio de San Juan está numerado del 1 al 23, mientras que no están numerados, y colocados aparte, por ser menos canónicos, la Carta a los Hebreos, las Epistolas de Santiago y Judas y el Libro de la Revelación; criterio que Tyndale modificó más tarde. En lo que concierne a la traducción, ésta se asienta fundamentalmente en Lutero. Así en el capítulo 2 de San Mateo, Lutero traduce: «Auff dem gebirge hat man ein jersey gehoret», y Tyndale sigue la misma dirección: «On the hills was a voice heard». Y mientras la versión griega, del capítulo 9 de San Mateo, dice Ten piedad de nosotros, hijo de David, Tyndale recoge: «Oh thou son of David have mercy on us», siguiendo directamente la frase de Lutero: «Ach du sohn David, erbarm dich unser». Además de los dos casos vistos, hay muchísimos más.

Por lo que se refiere a las notas al margen, buena parte son traducciones directas de Lutero. Así, hay más aquí en una página que en cualquier otro texto de Tyndale. Para darnos una idea, es conveniente recordar que el Nuevo Testamento de 1536, publicado en Worms, no tiene ninguna y que el Génesis, que vio la luz en 1530, tan sólo seis. Pues bien: sesenta de esas notas al margen son leves adaptaciones de Lutero y treinta corresponden a Tyndale, que son meras aclaraciones, como la que observamos en el capítulo 3 de San Mateo, «Locusts are more than our grasshoppers», las langostas son más grandes que nuestros saltamontes. Por otra parte, Tyndale elimina toda andanada 
antipapista o anticlerical de Lutero.

El Prólogo de Tyndale se basa ampliamente en el agustino alemán. Corresponde a su «Vorrhede» de su Nuevo Testamento de 1522. El autor inglés lo traduce, modifica, suprime párrafos, pero, sobre todo, añade. Las siete páginas de Lutero pasan a ser catorce en Tyndale, pues desarrolla la idea de en qué consisten los Testamentos Antiguo y Nuevo; qué significan las palabras «Evangelio»y «Ley», siguiendo a San Pablo; y qué es el pecado. Cuando Tyndale refleja sus propias ideas, se nos revela como un buen conocedor de San Pablo, además de un buen maestro de la teología neotestamentaria. Su Prólogo termina con cinco páginas que no encontramos en el Nuevo Testamento de Lutero, aunque son luteranas en el tono duro de exposición de nuestra esclavitud satánica. Nos llega a dar la impresión de que es una traducción fiel de algún texto luterano, que ni siquiera Tyndale habría traducido. Sin embargo, no se ha encontrado ese texto original. Emprender la tarea de su búsqueda en los cincuenta y ocho volúmenes de la edición Weimar de Lutero podría sobrepasar toda paciencia bíblica...

Cuando Tyndale expresa sus propias ideas, se basa en la Escritura, que llega a parafrasear. La impresión que nos da es que, cuando cita, lo hace de memoria. Aunque cabe que nos preguntemos: ¿qué es lo que cita de memoria? Porque no existía traducción alguna de San Pablo. Sería el propio Tyndale el autor de la primera. A este respecto conviene tener en cuenta tres cosas: con cierta frecuencia sus citas no corresponden con la traducción de 1526; parecen estar más en línea de las traducciones lolardas del manuscrito del inglés medio, realizadas bajo la dirección de Wyclif, aunque, a veces, tampoco parece ser el caso; por último, de lo que se desprende de las observaciones anteriores, podríamos encontrarnos ante la evidencia de la existencia de un conjunto de frases memorizadas que serían traducción de la versión del Nuevo Testamento, 
tomada del griego y que Erasmo publicó en 1516. Esas frases se utilizaron para la exposición en los sermones.

\section{V}

El primer Nuevo Testamento completo de Tyndale lo imprimió Peter Schoeffer en la pequeña y segura, ciudad luterana de Worms, entre 1525 y 1526. Es muy diferente del fragmento de Colonia. Es un libro en octavo. Carece de Prólogo y no tiene notas al margen. Es un texto sin adiciones, con pequeñas iniciales ilustradas al comienzo de cada libro. Al final, hay tres páginas «To the Reader» y otras tres de erratas.

Schoeffer no fue un impresor prolífico. Así, entre 1513 y 1526, tan sólo publicó cuatro libros, y se especializó en Biblias alemanas protestantes:

Schoeffer is said to have become a Lutheran, and on that account to have (...) settled at Worms. This is confirmed by several facts. The German bible which he printed is a Protestant version, and it is stated that it was suppressed on that account. He was denounced as a printer of heretical books, his name being in the list of printers from whose offices have issued works by heretics... 6 .

Cochlaeus informó de que este Nuevo Testamento tuvo una tirada de seis mil ejemplares. Las copias, a modo de contrabando, se introdujeron en Inglaterra en balas de paño, y la venta estuvo a cargo de «Master Garrett, Curate of All Hallows in Honey Lane London». Era febrero de 1526:

About the year of our Lord 1526, Master Garret, cuarate in Honey-lane, in London, came unto Oxford, and brought with him sundry books in Latin, treating of the Scripture, with the first part of "Unio dissidentium", and Tyndale's first translation of the New Testament in English; which books he sold to divers

6[William Tyndale], The First New Testament Printed in the English Language (1525 or 1526), Translated from the Greek by William Tyndale, Reproduced in Facsimile, with an Introduction by Francis Fry, Bristol, Printed for the Editor, 1862, pp. 8-9. 
scholars in Oxford, whose names, for his accountable memory, belike, he wrote in a small book of accounts?

El Obispo de Londres, presionado por el cardinal Wolsey, confiscó todos los ejemplares que pudo y los quemó delante de la iglesia de San Pablo. Para la ocasión, el prelado pronunció un sermón en el que afirmó que la traducción de Tyndale tenía más de dos mil errores (sic). Dicho en palabras más claras: Tyndale había traducido del griego, y demostrado, al menos a los ojos de algunos, la inferioridad del texto de la Vulgata. El obispo londinense, Cuthbert Tunstall, que había ayudado a Erasmo en la búsqueda de manuscritos para su Nuevo Testamento en griego, actuó con hipocresía, quizá superado por el gran poder de Wolsey:

For it was not unknown to cardinal Wolsey, and to the bishop of London, and to others of that ungodly generation, that Master Garret had a great number of those heretical books, as the world then accounted them; and that he was gone to Oxford, to make sale of them there, to such as he knew to be the lovers of the gospel. Wherefore they determined to make forthwith a privy search through all Oxford, to apprehend and imprison him, and to burn all and every his (...) books, and him too, if they could: so burning hot was the charity of these holy fathers 8 .

La versión de Tyndale, traducción del griego, es excelente. Tiene inspiración. La calidad extraordinaria de la lengua inglesa, su claridad y atemporalidad, la riqueza de la gama de efectos..., no tiene parangón en ningún otro autor inglés del momento. Miles de sus frases pasaron intactas a la Versión Autorizada del King James en 1611: «with men, this is imposible, but with God all things are posible»; «the powers that be»; «the last enemy that shall be destroyed is death»; «work out your own salvation with fear and trembling»; «it is a fearful thing to fall into the hands of the living God»; «behold, I stand at

${ }^{7}$ [John Foxe], The Acts and Monuments of John Foxe: A New and Complete Edition. With a Preliminary Dissertation by the Rev. George Townsend, Edited by the Rev. Stephen Reed Cattley, 8 vols., London, R.B. Seeley and W. Burnside, 1838, vol. V, p. 421.

8 [John Foxe], loc. cit. 
the door and knock»; «O wretched man that I am, who shall deliver me from this body of death?»; «For here we have no continuing city, but we seek one to come».

De esta edición de 1526 sólo se conservan dos ejemplares completos. Uno de ellos salió a la luz en 1996. En 1994, en el quinto centenario de Tyndale, la Biblioteca Británica adquirió el que se consideraba único, propiedad del Bristol Baptist College. Se dice que el importe de la venta ascendió a un millón de libras. Dos años más tarde, 1996, y casi por casualidad, apareció la copia de Stuttgart:

The story of this book up to today is miraculous. It escaped the mortal threat of Tyndale's prosecutors, as well as three great wars in the seventeenth and twentieth centuries; it moved from Protestant to Catholic hands to and fro. As far as it was accessible, nobody apparently appreciated its inner value. Each time when an English expert sought for a first Tyndale copy, it hid itself. Whe the supposedly unique Bristol copy was presented on its tour through America, suddenly its surviving brother slipped out of hiding to reveal itself to the world 9 .

La quema de las Biblias afectó mucho a Tyndale. Con este suceso comenzaron sus ataques a la jerarquía eclesiástica. Anteriormente ya se había lamentado de que no hubiera ningún lugar en Inglaterra en el que se pudiera imprimir una Biblia en inglés:

... in London [I] saw things whereof I defer to speak at this time and understood at the last not only that there was no room in my lord of London's palace to translate the new testament, but also that there was no place to do it in all England, as experience doth now openly declare ${ }^{10}$.

\footnotetext{
9 Eberhard Zwink, «Confusion About Tyndale: The Stuttgart Copy of the 1526 New Testament in English», Reformation, Vol. Three (1998), pp. 30-48; p. 48.

10David Daniell, ed., Tyndales's Old Testament, Being the Pentateuch of 1530, Joshua to 2 Chronicles of 1537, and Jonah, Translated by William Tyndale, New Haven \& London, Yale University Press, 1992, p. 5.
} 
Tyndale sabía, igualmente, que sus enemigos en la iglesia inglesa estaban dispuestos a condenarlo por hereje por pequeña que fuera la falta en la que lo pudieran sorprender:

... they which in times past were wont to look on no more scripture than they found in their Duns or such like devilish doctrine have yet now so narrowly looked on my translation, that there is not so much as one "i" therein if it lack a little over his head, but they have noted it, and number it unto the ignorant people for an heresy ${ }^{11}$.

Semejante destrucción, tan espectacular, tan coordinada, tan organizada por la iglesia inglesa para impedir que el pueblo llano leyera y comprendiera la Biblia fue una absoluta decepción para Tyndale.

\section{$[\mathbf{V I}$}

Después de la quema de sus Biblias, un hombre con menor entereza que Tyndale habría renunciado a seguir. Él no lo hizo. Aprendió hebreo. Y en enero de 1530 aparecieron en Inglaterra, procedentes de Amberes, ejemplares de The First Book of Moses Called Genesis. El Prólogo está encabezado con «W.T. to the Reader». Es la primera vez que Tyndale estampa su autoría en sus traducciones. El Génesis se podía comprar sólo o con los otros cuatro libros del Pentateuco. Cada uno dispone de su Prólogo y el Libro del Exodo cuenta con once grabados a toda página. El colofón dice: «Hans Luft at Marlborow», una dirección falsa, y necesaria, para imprimir esta clase de libros en aquel momento. Hasta fecha reciente, se creyó que se trataba de un tal Johannes Hoochstraden, de Amberes. Era tan solo pseudónimo del impresor habitual de las obras de Tyndale en Amberes, M. de Keyser. El verdadero Hans Luft de Wittenberg era el impresor

${ }^{11}$ David Daniell, ed., 1992, op. cit., p. 3. 
de Lutero. De Keyser también utilizó otras direcciones falsas, como «Adam Anonymous, Basel». Otros impresores, con no poco sentido del humor, fijaban su residencia en «Utopia» y, en el caso de obras claramente luteranas, en Roma, en la Plaza de San Pedro.

El Génesis debió sorprender a sus lectores, pues en lugar de «Fiat lux, et lux erat», que la iglesia había venido repitiendo, o de «Be made light, and made is light», que identificaban los que conocían el manuscrito lolardo, Tyndale nos ofrece «Let there be light and there was light» ${ }^{12}$.

Los Libros del Éxodo y del Deuteronomio, en inglés por primera vez, están impresos en caracteres romanos. Los cinco Prólogos, y el hecho de que los cinco libros estén impresos con caracteres distintos indica que se editaron de forma separada y conjunta. Cada una de las notas al margen -tan sólo seis en el Génesis- no son frecuentes. En el capítulo 32 del Libro del Éxodo leemos en la nota: «The pope's bull slayeth more than Aaron's calf, even an hundred thousand for one hair of them»13. En el Libro de los Números, capítulo 23, a la pregunta de Balam: «How shall I curse whom God curseth not and how shall I defy whom the Lord defieth not?» La nota de Tyndale responde: «The pope can tell you»14. En general, todas estas notas del Pentateuco, un total de 132, nos presentan a un Tyndale, buen conocedor del hebreo, aclarando conceptos.

Estudiar el Pentateuco de Tyndale, auxiliados de una Biblia en hebreo y cotejando la antigua traducción griega, conocida como Septuaginta, la versión alemana de Lutero y la Vulgata, es sinónimo de contemplar al Tyndale traductor descubriendo significados y dándoles sentido y claridad en inglés. Se ha dicho que Tyndale no sabía griego y que se limitó a traducir a Lutero al inglés. En realidad, se sigue diciendo. Si bien, resulta ser más cierto que

12 David Daniell, ed., 1992, op. cit., p. 15.

${ }^{13}$ David Daniell, ed., 1992, loc. cit., p. 133.

${ }^{14}$ David Daniell, ed., 1992, loc. cit., p. 234. 
Tyndale traduce del hebreo de modo brillante. Es verdad que conocía, y dominaba, la traducción de Lutero del hebreo, y que la utilizó en aspectos referentes a gramática y vocabulario, lo mismo que hizo con la Septuaginta. Tyndale pensaba que el hebreo se adaptaba muy bien al inglés y así lo expresa, casi con vehemencia, en su Obedience of a Christian Man, de 1528:

And the properties of the Hebrew tongue agreeth a thousand times more with the English than with the Latin. The manner of speaking is both one, so that in a thousand places thou needest not but to translate it into the English word for word, when thou must seek a compass in the Latin, and yet shall have much work to translate it well-favouredly, so that it have the same grace and sweetness, sense and pure understanding with it in the Latin, as it hath in the Hebrew. A thousand parts may it be translated into the English, than into the Latin. Yea, and except my memory fail me, and that I have forgotten what I read when I was a child, thou shalt find in the English chronicle, how that king Aldestone caused the holy scripture to be translated into the tongue that then was in England, and how the prelates exhorted him thereto ${ }^{15}$.

Tyndale entendió la gama y variedades de la escritura hebrea y la vertió al inglés con un vigor y fortaleza que, todavía hoy, casi 500 años después, se aprecian. Así, en el capítulo 3 del Génesis comienza diciendo:

But the serpent was subtler than all the beasts of the field which the Lord God had made, and said unto the woman. Ah sir, that God hath said, ye shall not eat of all manner trees in the garden. And the woman said unto the serpent, of the fruit of the trees in the garden, we may eat, but of the fruit of the tree that is in the midts of the garden (said God) see that ye eat not, and see that ye touch it not: lest ye die.

Then said the serpent unto the woman: tush ye shall not die: But God doth know, that whensoever ye should eat of it, your eyes should be opened and ye should be as God and know both good and evil. And the woman saw that it was a good tree to eat of and lusty unto the eyes and a pleasant tree for to make wise. And took of the fruit of it and ate, and gave unto her husband also with her, and he ate. And the eyes of both of them were opened, that they understood how that they were naked. Then they sewed fig leaves together and made them aprons ${ }^{16}$.

15 [William Tyndale], Doctrinal Treatises and Introductions to Different Portions of the Holy Scriptures, By William Tyndale, Martyr 1536, Ed. by Henry Walter, Cambridge, Printed at the University Press for the Parker Society, 1848, pp. 148-9.

16David Daniell, ed., 1992, op. cit., p. 17. 
Cada Prólogo del Pentateuco de Tyndale tiene su interés. El primero nos ofrece algunos datos autobiográficos y las razones que le condujeron a abandonar Gloucester y, posteriormente, Inglaterra. El Prólogo del Deuteronomio comienza así:

This is a book worthy to be read in day and night and never to be out of hands. For it is the most excellent of all the books of Moses. It is easy also and light and a very pure gospel that is to wete, a preaching of faith and love: deducing the love to God out of faith, and the love of a man's neighbour out of the love of God. Herein also thou mayst learn right meditation or contemplation, which is nothing else save the calling to mind and a repeating in the heart of the glorious and wonderful deeds of God, and of his terrible handling of his enemies and merciful entreating of them that come when he calleth them, which thing this book doth and almost nothing else ${ }^{17}$.

Aquí tenemos las frases características de Tyndale de expresión clara, sencilla y directa.

\section{[VII]}

Antes y después de publicar el Pentateuco en 1530, Tyndale sacó a la luz cinco monografías doctrinales, entre las que se cuentan algunos de sus libros que, todavía hoy, son poco conocidos, como The Parable of the Wicked Mammon y The Obedience of a Christian Man, ambos impresos, en 1528, en el taller de de Keyser; o la Answer que se ocupa de responder a lo que el Canciller Tomás Moro había escrito de él. Todos se conservan en ejemplares en buen estado. La ampliación de las reflexiones de Lutero sobre la Carta a los Romanos, la Compendious Introduction, que imprimió Peter Schoeffer en Worms, se conserva en una única copia en la Bodleian Library de Oxford. Su traducción de la Profecía de Jonás, a la que añadió un extenso prólogo, varias veces superior al

${ }^{17}$ David Daniell, ed., 1992, op. cit., p. 284. 
texto en sí, se conserva también en una única copia, rescatada a finales del siglo XIX, y hoy depositada en la Biblioteca Británica. Se imprimió en Amberes en el taller de de Keyser. Por último su exposición del Sermón de la Montaña y las tres Epístolas de San Juan se imprimieron en Amberes por de Keyser.

La revisión que Tyndale realizó de su Nuevo Testamento se imprimió en noviembre de 1534 por Martin el Emperador, es decir: de Keyser. Este librito es el más importante de todos los publicados por de Keyser y, puede, que por todos los impresores de Amberes, en la década de 1530. Es el verdadero predecesor de la Biblia inglesa y, afortunadamente, se conserva un número razonable de ejemplares. Uno de ellos, impreso en vitela, perteneció a la reina Ana Bolena. Dispone de anotaciones personales y se conserva, con otros, en la Biblioteca Británica.

En este caso, existen dos Prólogos extensos al Nuevo Testamento. El primero empieza relatando el descubrimiento de Tyndale de la presencia de la lengua hebrea en el Nuevo Testamento en griego. Está impreso en letra gótica. Todos los libros disponen de un Prólogo que, en ocasiones, siguen la senda de los de Lutero. El de la Carta a los Romanos es de una extensión excepcional. Resulta ser traducción del Prólogo de Lutero, con párrafos que añade Tyndale, que lo convierte en un tratado tan largo como la propia epístola de San Pablo. Se trata de una argumentación densa sobre la importancia de esta carta:

Forasmuch as this epistle is the principal and most excellent part of the new testament, and most pure evangelion, that is to say glad tidings and that we call gospel, and also a light and a way in unto the whole scripture, I think it meet, that every Christian man not only know it by rote and without the book, but also exercice himself therein evermore continually, as with the daily bread of the soul ${ }^{18}$.

${ }^{18}$ David Daniell, ed., Tyndale's New Testament, Translated from the Greek by William Tyndale in 1534, New Haven and London, Yale University Press, 1989, p. 207. 
En lo que se refiere a la erudición, Tyndale manifiesta su independencia. A diferencia de Lutero, evita condenar cualquier parte de la escritura de la versión griega. Respecto a la Carta a los Hebreos, de la que siempre se han tenido dudas de que sea de San Pablo, Tyndale se muestra más condescendiente que Lutero.

Los únicos libros que no disponen de Prólogo son los Hechos de los Apóstoles y el de la Revelación, que posee veintitrés huecograbados, muy similares a los que hallamos en el segundo Nuevo Testamento de Lutero, de 1522; el denominado de Diciembre. A lo largo del Nuevo Testamento de Tyndale hay frecuentes notas al margen, todas ellas breves y aclaratorias. La revisión a la que sometió al Nuevo Testamento de 1526 es minuciosa. Tyndale es un traductor profesional que revisa constantemente, que modifica, que considera cualquier detalle, venga de donde venga, y no digamos de su recién adquirido conocimiento del hebreo. Entre lo mucho que todavía queda por hacer con el legado de Tyndale, hay algo destacable: el estudio de las fuentes utilizadas en sus revisiones. Se sirve de los textos, en paralelo, griegos y latinos de Erasmo; del Nuevo Testamento de Lutero, de la traducción francesa del Nuevo Testamento, obra de Le Fèvre, y de las Escrituras en hebreo. Hay que lamentar que Tyndale no viviera para traducir los libros poéticos y las profecías del Antiguo Testamento, pues su obra completa sobrepasaría, con mucho, la de Miles Coverdale, que fue la que se adoptó. Y lo que hubiera resultado más importante: se habría logrado una mejor sintonía con el Nuevo Testamento que, en buena parte, está entrelazado con esos libros y profecías. Pero fue encarcelado y conducido a una muerte segura por las argucias mezquinas de un villano. Dicho de otra forma: es como si William Shakespeare hubiera sido asesinado por un Yago real, a la mitad de su vida, y no hubiera podido escribir sus celebradas tragedias.

Afortunadamente, tenemos el Nuevo Testamento de 1534 que, en un 
ochenta por ciento, es el Nuevo Testamento que los angloparlantes han conocido hasta fechas muy recientes. Dice Tyndale en el capítulo 2 del Evangelio de San Lucas:

And there were in the same region shepherds abiding in the field and watching their flock by night. And lo: the angel of the Lord stood hard by them, and the brightness of the Lord shone round them, and they were sore afraid. For behold, I bring you tidings of great joy that shall come to all the people: for unto you is born this day in the city of David, a saviour which is Christ the Lord. And take this as a sign: ye shall find the child swaddled and laid in a manger. And straightway there was with the angel a multitude of heavenly soldiers, lauding God and saying: Glory to God on high, and peace on the earth: and unto men rejoicing ${ }^{19}$.

Y el capítulo 14 del Evangelio de San Juan empieza diciendo:

+ And he said unto his disciples: Let not your hearts be troubled. Believe in God and believe in me. In my father's house are many mansions. If it were not so, I would have told you. I go to prepare a place for you. And if I go to prepare a place for you, I will come again, and receive you even unto myself, that where I am, there may ye be also. And wither I go ye know, and the way ye know ${ }^{20}$.

\section{VIII}

Los impresores de Amberes, durante treinta años, hicieron buena caja a cuenta de suministrar Biblias al lector inglés. Una tirada de 3.000 ejemplares, fue solo el comienzo, aun cuando el libro estaba prohibido. Pero eso no fue todo. El segundo Prólogo a la revisión del Nuevo Testamento de 1534 lleva el título de «W.T. yet once more unto the Christian Reader» y constituye una larga recriminación a su antiguo ayudante George Joye, al que habían convencido para que fuera corrector de pruebas de ediciones piratas del Nuevo Testamento de Tyndale publicado en Worms en 1526. La impresora, en este caso, iba a ser la

${ }_{19}$ David Daniell, ed., 1989, op. cit., p. 91.

${ }^{20}$ David Daniell, ed., 1989, op. cit., p. 154. 
viuda de Christopher van Endhoven, al que habían encarcelado en Londres, en 1531, por vender ejemplares del Nuevo Testamento de Tyndale, donde murió en prisión. Joye aceptó el encargo. Van Endhoven imprimió y vendió tres ediciones piratas en 1527, 1530 y 1533/4. Por impresiones posteriores sabemos que fueron sextodécimos raros, pequeños y mal encuadernados. Pero van Endhoven vendió 7.000 ejemplares, lo que es buen indicativo del mercado de lectores británicos. Con dos ediciones piratas posteriores y la revisión de Tyndale de 1534, llegamos a un total de 16.000 ejemplares en circulación antes de finalizar 1534.

Sin embargo, Joye hizo algo más que corregir pruebas. Aunque nos pueda parecer una locura, alteró la traducción de Tyndale, cambiando, por ejemplo, «resurrection» por «life after life», aspecto de mucha sensibilidad en aquel momento. Tyndale es rotundo en su reproche y dice que Joye puede modificar el texto cuantas veces quiera, siempre y cuando ponga su nombre. Tyndale pone sus iniciales en su edición, reprende a Joye, dice a los lectores cuál es su edición y se olvida del asunto. A lo que parece, Joye continuó con la disputa.

Después de esto, nos encontramos a Tyndale trabajando en el Antiguo Testamento, en la parte que comprende los libros históricos, desde el de Josué hasta el Segundo de las Crónicas. Aquí sí que precisa superior conocimiento de hebreo y de inglés. Nadie, en 1535, utilizaba el inglés con semejante gama de estilos: desde el himno de victoria más antiguo en la "Canción de Debora", en el capítulo 5 del Libro de los Jueces, pasando por algunos salmos, hasta llegar a las narraciones largas de intrigas en la corte, en los Libros de Samuel y de los Reyes, las hazañas épicas de David y luego, Elías. Podemos, incluso, llegar a percibir los tonos dramáticos del capítulo 18 en el Primer Libro de los Reyes:

And Ahab went against Eliah. And when Ahab saw Eliah, he said unto him: art thou he that troubleth Israel? And he said: it is not I that trouble Israel, but thou and thy father's house, in that ye have forsaken the commandments of the Lord 
and hast followed Baal. But now send and gather to me all Israel unto mount Carmel and the prophets of Baal four hundred and fifty, and the prophets of the groves four hundred, which eat of Jezebel's table. And Ahab sent for all the children of Israel, and gathered the prophets unto mount Carmel.

And Eliah came unto all the people and said: why halt ye between two opinions? If the Lord be very God, follow him: or if Baal be follow him. And the people, answered him not one word ${ }^{21}$.

Nos encontramos ante una lengua inglesa prácticamente desconocida en la década de 1530; un uso dramático del estilo de prosa llana que Tyndale concedió a Inglaterra, para su disfrute durante dos siglos y medio; hasta que Samuel Jonson preconizó los modelos latinos.

[IX

Tyndale convivió en la Casa Inglesa de Amberes con mercaderes ingleses 22 . La Casa Inglesa le garantizaba su seguridad, siempre que estuviera en sus dependencias. En mayo de 1535, un inglés, de nombre Henry Phillips, y probablemente a sueldo de John Stokesley, sucesor de Tunstall en la sede episcopal de Londres, que había estudiado sus movimientos, lo sorprendió en una de sus salidas. Tyndale fue arrestado. Pasó dieciséis meses en una mazmorra del castillo de Vilvoorde, a las afueras de Bruselas. Un miembro de la Casa Inglesa, Thomas Poyntz, que tenía buenos contactos en la corte inglesa, puso todo su empeño para que fuera liberado, poniendo en peligro su negocio,

${ }^{21}$ David Daniell, ed., 1994, op. cit., p. 492.

22 Amberes era un centro neurálgico para el comercio inglés. La presencia de estos mercaderes la certifica la Compañía de los Merchants Adventurers, corporación gremial que contaba con cédula real y que contaba entre sus filas a lo más destacado del comercio inglés. En términos contables son de destacar John Weddington, factor de Sir Thomas Gresham, figura de gran relevancia en el reinado de Isabel I. El primero, en 1567, dio a la imprenta uno de los tratados contables más pertinentes que se publicaron durante la dinastía Tudor. Resulta curioso cómo, a fin de cuentas, lo material y lo espiritual convergieron en aquel punto estratégico del comercio europeo. 
vida familiar y el favor de la corte. Estaba a punto de conseguirlo cuando Phillips lo arrestó por hereje luterano. Tyndale, juzgado y acusado de herejía, fue desposeído de su orden sacerdotal y entregado a las autoridades civiles que lo quemaron en la hoguera ${ }^{23}$. Se cuenta que sus últimas palabras fueron: «Lord, open the King of England's eyes». Nunca han estado claras las razones que le condujeron a la muerte, salvo la avidez incontrolada de dinero de Phillips y la maldad del obispo Stokesley que, en su lecho de muerte, se declaró responsable de haber enviado a la hoguera a treinta y cinco personas.

Pocos meses después del martirio de Tyndale en 1536, John Rogers, capellán de la Casa Inglesa en Amberes, y que más tarde también moriría en la hoguera, durante el mandato de la reina Mary, reunió todas las traducciones de Tyndale, incluidos los libros históricos no publicados, y los imprimió en un folio elegante, con el dinero de dos impresores londinenses: Grafton y Withchurch. Ignoramos dónde se imprimió. Probablemente fue en Amberes. La primera página lleva impresa una ironía sangrante: «Set forth with the King's most gracious licence». Paradojas de la historia. Si Tyndale hubiera vivido unos meses más, habría visto que los ojos del monarca, comenzaban a abrirse.

Roger no podía recoger el nombre de Tyndale en la publicación, por lo que se dice que la Biblia la edita «Thomas Matthew», dos nombres de dos de sus discípulos. Es un volumen con excelentes grabados y seis iniciales minuciosamente decoradas. Las que se encuentran entre los Profetas y los Apócrifos son «W T». La identidad del traductor no era un secreto para nadie, si acaso, para Enrique VIII. La mitad del Antiguo Testamento que Tyndale no pudo traducir -los libros poéticos y proféticos-, Roger los toma de la Biblia de Coverdale, publicada en 1535. Por primera vez se tenía una Biblia impresa en ${ }^{23}$ Brian Moynahan, William Tyndale: If God Spare My Life. A Store of Martyrdom, Betrayal and the
English Bible, London, Abacus, 2003. 
inglés, aunque en la parte añadida decrece la erudición; lo que se aprecia, sobre todo, en los Salmos. Los que se imprimieron en el Book of Common Prayer de 1549 y las subsiguientes ediciones hasta llegar a 1960, son de la Biblia de Coverdale de 1535. En ocasiones poseen cierta belleza en la frase, pero en otras, son incomprensibles, lo que nunca sucede en Tyndale. Su patente es siempre la claridad.

La obra de Tyndale, desde esa edición de "Thomas Matthew" de 1537, pasó a la edición revisada de Coverdale de 1539, la denominada Great Bible y de ésta a la Geneva Bible de 1560 y sus ediciones posteriores. Así hasta llegar a la mesa de los traductores designados por el rey James en 1607 que la adoptaran en su mayor parte. De esta suerte, la King James Bible, durante cuatro siglos, ha sido, sin decirse, obra de Tyndale.

$[X]$

Se suele decir que la King James Bible es magnífica porque se tradujo en un momento en el que el inglés, que había salido prácticamente de la nada, de las décadas de 1520 y 1530, había alcanzado la gloria de todo su esplendor, como lo demuestra la obra de Shakespeare. En ocasiones se ha extendido la especie de que el rey designó al dramaturgo para que le diera un toque poético a su versión. Resulta ser una aseveración incierta, además de desafortunada desde cualquier interpretación.

En el primer tercio del siglo XVI, de 1500 a 1530, la inglesa era una lengua pobre, aventajada por el nuevo latín humanista. En algunos ámbitos de la vida inglesa, como testamentos y libros parroquiales, se empezaba a usar el inglés, pero nada extraordinario. El inglés no era el medio de transmisión para ningún asunto de cierto empaque. En el siguiente tercio, la expresividad de la lengua 
aumentó de forma notable. Y superó con creces al latín en el último tercio. En la última década los recursos de la lengua inglesa crecieron de tal forma que permitió a los traductores del rey servirse de una lengua rica.

$\mathrm{Y}$ aunque los traductores reales hicieron en toda la Biblia varios miles de cambios, el Nuevo Testamento que produjeron, y la mitad del Antiguo, son de Tyndale en vocabulario, sintaxis y sobre todo, cadencia. Y los escribió cuando el inglés era poca cosa: ochenta años antes del King James y sesenta antes de Shakespeare.

Tyndale entregó la vida, en ambos sentidos, para acercar al pueblo llano la palabra de Dios. Todavía hoy es un lenguaje que llega al corazón. Así concluye el capítulo 14 de San Lucas, en la versión de Tyndale, y nosotros con él:

And he said unto him: Son thou was ever with me, and all that I have, is thine: it was meet that we should make merry and be glad: for this thy brother was dead, and is alive again: and was lost, and is found ${ }^{24}$.

24David Daniell, ed., 1989, op. cit., p. 117. 